\title{
Spectrogram Enhancement By Edge Detection Approach Applied To Bioacoustics Calls Classification
}

\author{
W.B. Hussein", M.A. Hussein and T. Becker \\ Group of (Bio-) Process Technology and Process Analysis, Faculty of Life Science \\ Engineering, Technische Universität München, Germany \\ whusseinewzw. tum.de
}

\begin{abstract}
Accurate recognition of sound patterns in spectrograms is important step for further recognition applications. However, background noise forms fundamental problem regardless the species under study. In this paper, crest factor feature was extracted from the limited dynamic range spectrogram. The developed crest factor image behaved as smoothed version of the spectrogram, at which edges of the involved sound patterns were detected without the need of prior smoothing filters and their scaling constraints. Attached noise - surrounds the detected edges - was removed, to form the enhanced spectrogram. The method was compared to other enhancement approaches such like spectral Subtraction and wavelet packet decomposition. Comparison was performed on different structure patterns of bats and birds. Results indicate how the method is promising for efficiently enhancing the spectrogram while preserving its temporal and spectral accuracy. The method correctly classified three bioacoustics species with an accuracy of $94.59 \%$, using few $2 D$ features of their enhanced spectrograms
\end{abstract}

\section{Keywords}

Spectrogram Enhancement, Edge Detection, Bioacoustics Classification, Pattern Recognition.

\section{Introduction}

\subsection{Problem formulation}

Bioacoustics calls have been efficiently employed for a long time for species detection, classification, and recognition. These calls handle the sound patterns which are almost unique and oriented for the investigated bio-source. Several temporal and spectral sound features are extracted from these patterns in time and frequency domains; respectively. These features are used to train and develop a learning system, using methods such as Artificial Neural Network, which afterwards is able to successfully recognize the test bioacoustics calls to their corresponding groups or species, according to their features values. The approach have been widely used in many life science problems, such as the bioacoustics detection of hidden grain weevils for early treatment [1], and detection of bat ultrasound echolocation calls in the windmills region to avoid their expected collision with the blades[2].

In general, collection of time domain and frequency domain features are used to develop more accurate detection system, revealing the importance of having a reliable spectrogram (timefrequency intensity 2D image) of the specified sound.Spectrogram is an important representation of sound data looks like the human hearing which is based on a kind of real-time spectrogram 
Signal \& Image Processing : An International Journal (SIPIJ) Vol.3, No.2, April 2012

encoded by the cochlea of the inner ear [3], to classify and recognize patterns of sound samples. However, spectrogram is usually attached by different forms of noise; including those formed during sound recording[4], and those produced during the transformation to frequency domain result in spectral leakage, and up to $10 \%$ error(s) infrequency and/or power spectrum computations [5]. These noises directly degrade the quality of the waveform, deteriorate the worth of the extracted features and thus lead to inaccuracy in recognition of the sound patterns [6].Further difficulty is added to the problem due to variability of patterns structure, which can vary greatly including vertical straight, sloped straight, sinusoidal type and relatively random patterns. However, filtering noisy signals through the spectrogram is considered more effective than separate filtrations in time and/or frequency domain, since sound patterns do not cover the whole spectrogram image, and therefore easier to filter off the noise.

A spectrogram enhancement approach which is independent on the noise type, level, and structure is required. Once established, the pattern recognition algorithms can operate efficiently and smoothly on the clear "only patterns" spectrogram. Therefore, the problem of spectrogram enhancement and accurate detection of the sound patterns has attracted researchers' interest from a variety of backgrounds ranging among signal and image processing, and statistical models $[7,8]$.

\subsection{Related work}

Common and recent techniques for spectrogram enhancement include basic band pass filtering[9], spectral subtraction[10], Wiener filter[11], and wavelet packet decomposition[12, 13].Simple methods, such as the band pass filtering, originally employed the use of time-domain filtering of the corrupted signal, however, this is only successful when removing low or high frequency noise and does not provide satisfactory results for many species which have frequency range overlaps with their attached noises [14].

Although the base spectral subtraction method is very simple and efficient, it assumes the noise to be additive and uncorrelated with the signal [15]. Moreover, the enhancement by spectral subtraction tends to produce sounds with musical artifacts that are often more objectionable than the original noise [16]. Later, the multi-band spectral subtraction method was proposed, at which the corrupted sound is initially divided into several frequency bands, and then the spectral subtraction method is applied to each band[17]. This method outperforms the standard power spectral subtraction method resulting in superior spectrogram quality and largely reduced musical noise. Meanwhile, the Wiener filter technique basically considers the beginning of a signal is noise, and its adaptive type removes noise based on a training data [18]. However, during the operation on data with unknown noise, the noise level can be underestimated and the enhancement can be slightly milder [19]. The methods based on wavelet packet decomposition are effective in removing background noise in the spectrogram. But they cannot suppress much of the noise generated during the Fourier transformation, because the former noise is usually random Gaussian distribution while the latter may be modeled by Rayleigh distribution [7,20].

Image analysis techniques applied to this area treat the spectrogram as an image, provides a wide range of methods which could be beneficial to this problem. One of these developed methods is the noise suppression using spectrogram morphological filtering [21,22], applying two subsequent operations of erosion and dilation. The erosion was responsible to remove noise from the noisy spectrogram while dilation used to restore any erroneously removed sound patterns. However, it improves the enhancement accuracy by only $10 \%$ when combined by nonlinear spectral subtraction with a suitable selection of the threshold. The author in [23] proposed an edge detection method which initially smoothes the spectrogram using a Gaussian filter, followed by thresholding each point by comparison to the background measurement. This allows for time invariant noise conditions and computing independently for each frequency bin, which successfully detected $(90 \%)$ of whale calls. If the smoothing kernel is quite large, the detrimental effect is reduction in the detection accuracy, especially at low SNR. Meanwhile, the authors in [24] passed the spectrogram through 2D bilateral filter to reduce noise and preserve its patterns 
edges. The filtered spectrogram is sent to two parallel processing paths, at which the first path extracts significant patterns from background noise. The second path performs the edge detection and restructures the rough patterns that can be used as a mask. The processed image from the first path passes through a mask generated from the second path. However, pattern recognition still severely depends on image processing skills and spectrogram resolutions which lead to concealment of very short patterns. Apartial differential equation technique was used in [25] for edge enhancement and noise reduction based on a regularization of the mean curvature motion equation. However, the comparisons indicate that the method gives almost similar results as the wavelet based methods.

In this paper, an improved spectrogram enhancement method has been developed based on the last advances in the edge detection techniques. The dynamic power range of the spectrogram is limited to avoid the problem of low level portions of the spectrogram expanding and thereby obscuring the detail of the energetic portions. Afterwards, the crest factor image is calculated as a smoothed version of the original spectrogram image, hence escaping the application of smoothing Gaussian filters and their drawbacks [26].Based on the edge detection algorithm presented in [28], the sound patterns in the crest factor image are detected. Afterwards, the original power values of the patterns edges and their interior are reconstructed, while the power values of the patterns surrounding are eliminated, as they represent the attached noises whether attached to the sound or generated during the frequency domain transformation. The proposed method was applied to several bioacoustics calls of different SNR values, and compared to the results given by band pass, multi-band spectral subtraction, Wienerfilter, and wavelet packet decomposition methods, with respect to subjective and objective measures. Finally, possible implementations of the proposed method in obtaining the enhanced frequency and power contours, reconstruction of the enhanced waveform, and simplified pattern recognition operation are presented.

\section{Material and method}

\subsection{Signal processing}

Audio sound streams are sampled in time domain with suitable sampling frequencies, selected to be higher than the double of maximum frequency in the sound stream, satisfying the Nyquist sampling theorem [5] and avoid antialiasing in the signal reconstruction. Figure (1) shows an example for the call of Sitta canadensis bird which was sampled at $11025 \mathrm{~Hz}$. The signal is divided into segments with length of $1 \%$ of the total signal length and $90 \%$ overlapping percentage. Each segment is then multiplied by Bartlett window function and transformed to frequency domain through Fast Fourier Transform (FFT). The frequency domain representation of the signal (i.e., spectrogram) is the power spectrum distribution with frequency, at each time instant, as plotted in figure (2).The implementation of the Bartlett window function is to have better frequency resolution while keeping acceptable spectral leakage and amplitude accuracy [27].

The resultant spectrogram contains important sound patterns of the signal immersed in attached noise. These noises are not only due to the base noise attached to the sound, but also generated during FFT, therefore, cleaning the signal in time domain, will not ensure clean spectrogram. 
Signal \& Image Processing : An International Journal (SIPIJ) Vol.3, No.2, April 2012

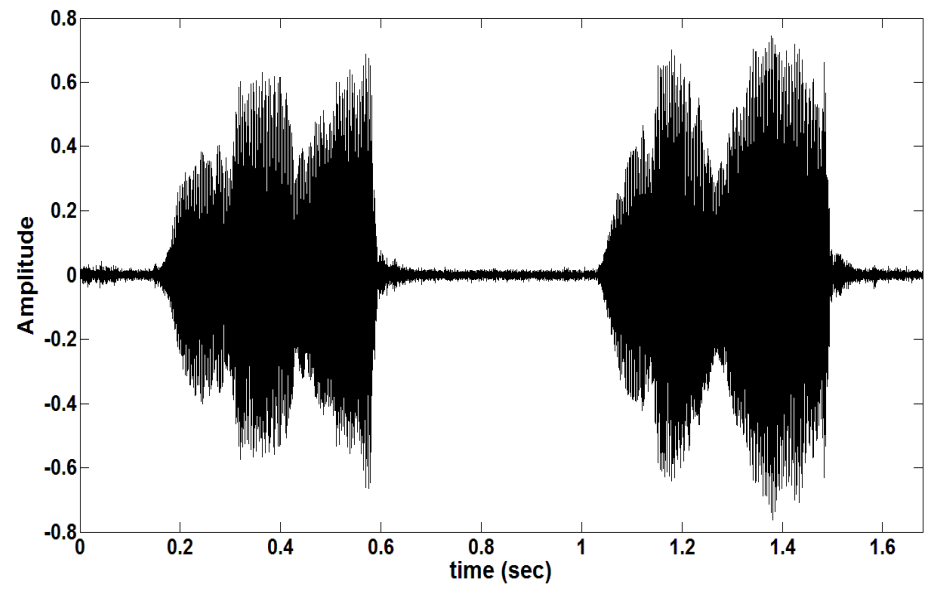

Figure 1. A sound stream for Sitta canadensis bird in time domain revealing its contents of two long pulses and one long inter-interval.

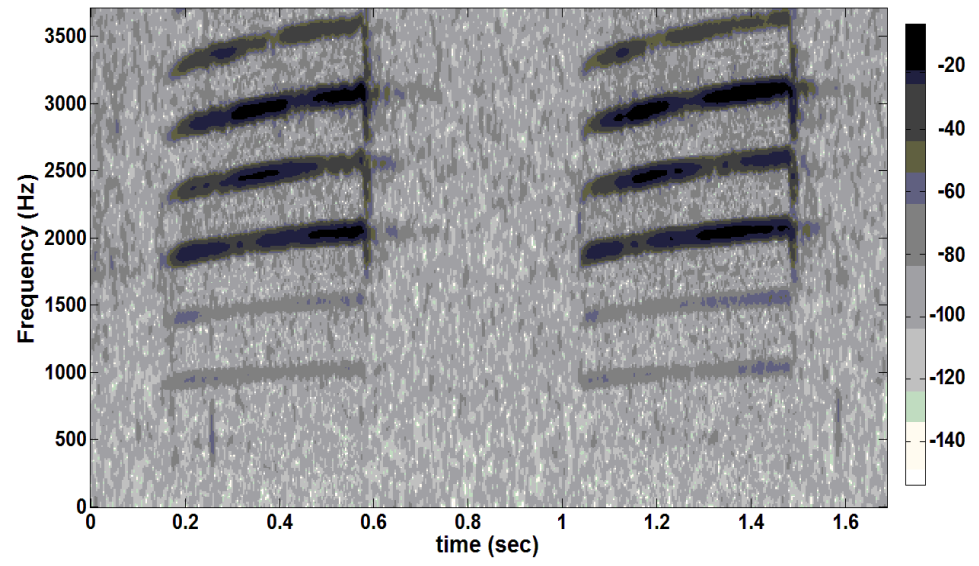

Figure 2. The spectrogram for the time domain signal of figure (1), after being divided into segments of length equal to $1 \%$ of the total signal length, multiplied by Bartlett window function and transformed to frequency domain using FFT.

\subsection{Limiting the dynamic range}

The attached noise to the spectrogram may be assumed to have almost same power value, which can be removed from the whole spectrogram. However, this will eliminate as well the non-noisy patterns which have this power value.Therefore, this thresholding scheme should be carefully applied through the physical fact of the limited dynamic range. Althoughthe whisper cannot be heard in loud surroundings, the spectrogram will contain all details about whisper and loud sound powers. Thus, the spectrogram powers have to be limited, to avoid much of the attached whisper (i.e., noise).The range is limited to $40 \mathrm{~dB}$ below the maximum value for all tested sounds, because most bioacoustics signals are expanded/slowed to the human speech range, which is normally perceived over this range [29]. Therefore, any point with power value outside this range, including those of noise as well as very weak patterns, are eliminated from the spectrogram, as shown in figure (3). 
Signal \& Image Processing : An International Journal (SIPIJ) Vol.3, No.2, April 2012

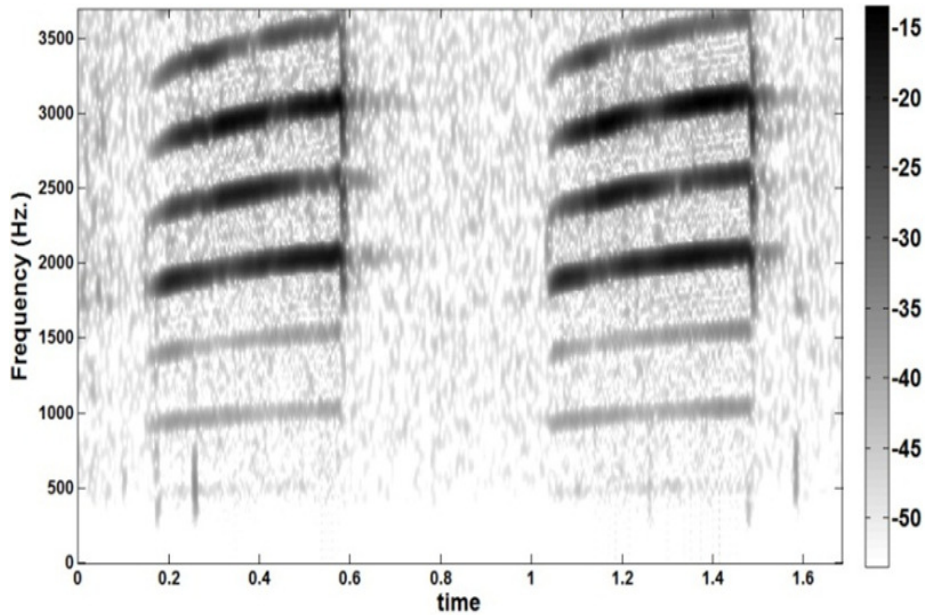

Figure 3. The limited spectrogram after the power values were limited to a dynamic range of $40 \mathrm{~dB}$, clarifying how most of the noise and very weak sound patterns were eliminated.

\subsection{Detection of the pattern edges}

The algorithm starts by sliding a $5 * 5$ matrix (mask) over the limited dynamic range spectrogram image in $x$ direction and then in $y$ direction, with step of one pixel. The represented pixel of the mask is its centroid which is calculated by equation (1). The intensities of the pixels (i.e., power values) enclosed by the mask are used to calculate the crest factor feature, given in equation (2), which is a ratio of the maximum value to the root mean square value, indicating how much impacting is occurring inside the mask, as schematically explained in figure (4).

$$
\begin{gathered}
\text { mask centroid }=\sum_{i=1}^{n} i * f_{i} \backslash \sum_{i=1}^{n} f_{i} \\
C=\frac{f_{\text {peak }}}{f_{r m s}}
\end{gathered}
$$

Where $f_{i}$ is the gray level intensity value of pixel $i$, and the back slash means that only the quotient of the division is considered. $f_{\text {peak }}$ and $f_{r m s}$ are the peak and root mean square of the pixels intensities; respectively, and $C$ is the crest factor of the mask.

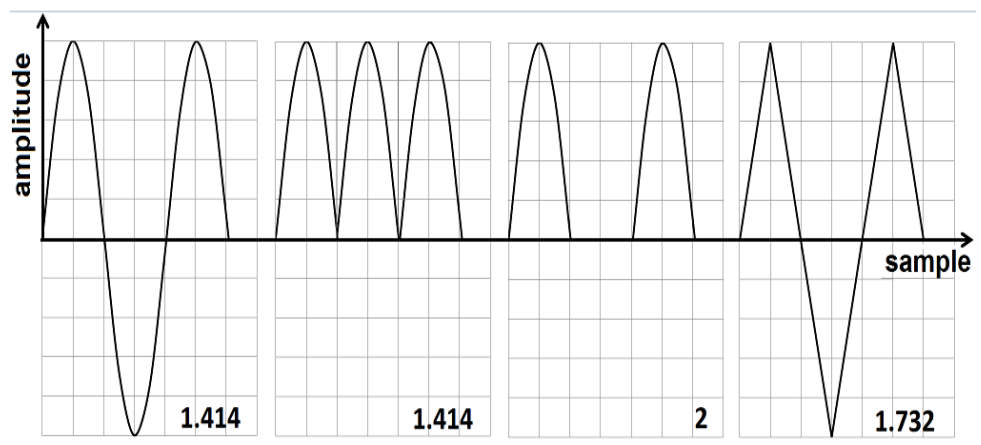

Figure 4. The crest factor for some basic curves, showing how much impacting occurs.

As a result, the crest factor image is obtained, by gathering the local crest factors calculated during the sliding of the mask. Although this edge detection algorithm follows the one presented in [28], it is applied to the crest factor image instead of combination of energy and skewness images, because this combination presents both strong edges (output of the energy feature) and weak edges (output of the skewness feature). Hence, the noises are also detected as patterns, displayed in figure (5a)with a signal to noise ratio (SNR) - given in equation (3) - of 1.95. Alternatively, the crest factor feature of an image presents the edges that have impact to their 
surrounding (i.e., non-weak edges), as displayed in figure (5b) with SNR of 2.82. Furthermore, the limited dynamic range makes the crest factor more meaningful since it is a measure of relative spatial intensity change.

$$
S N R=10 \log _{10} \frac{\sum P_{\text {clean }}}{\sum\left|P_{\text {clean }}-P_{\text {output }}\right|}
$$

Where $\mathrm{P}_{\text {clean }}$ and $\mathrm{P}_{\text {output }}$ are the clean and output power values; respectively.

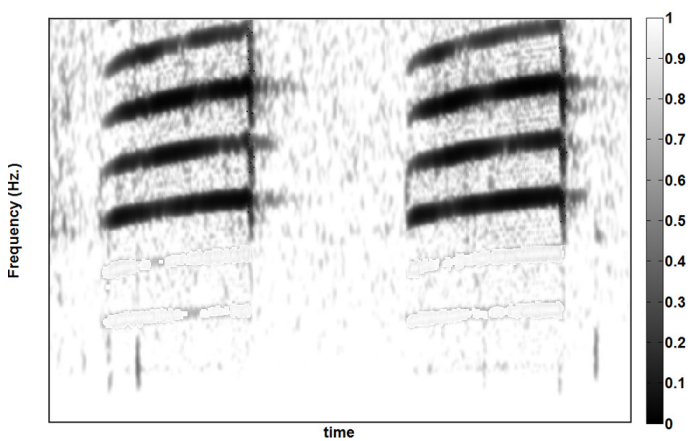

(a)

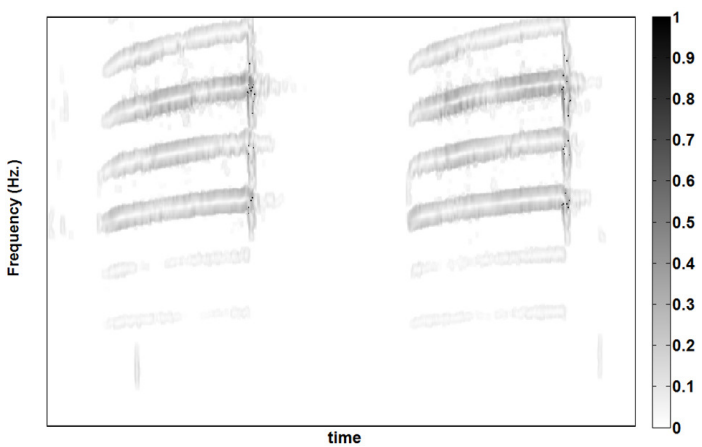

(b)

Figure5. Two features from the limited dynamic range spectrogram of figure (3), (a) combination of energy and skewness images, following the algorithm presented in [28], and (b) the crest factor image.

Afterwards, the Sobel operators given in equation (4) are employed on the crest factor image, through equation (5),to get its gradient images $\left(C_{x}\right.$ and $\left.C_{y}\right)$. And with the aid of equations (6) and (7), the edges strength (E) and the edges directions $(\theta)$ are calculated; respectively.

$$
\begin{gathered}
\Delta_{x}=\left[\begin{array}{ccc}
-1 & 0 & 1 \\
-2 & 0 & 2 \\
-1 & 0 & 1
\end{array}\right], \Delta_{y}=\left[\begin{array}{ccc}
1 & 2 & 1 \\
0 & 0 & 0 \\
-1 & -2 & -1
\end{array}\right] \\
C_{x}=\Delta_{x} * C, C_{y}=\Delta_{y} * C \\
E=\sqrt{C_{x}^{2}+C_{y}^{2}}(6) \\
\theta=\tan ^{-1}\left(\frac{C_{y}}{C_{x}}\right)+\frac{\pi}{2}
\end{gathered}
$$

Where $\Delta_{x}$ and $\Delta_{y}$ are the derivative operators in x and y directions; respectively. $C_{x}$ and $C_{y}$ are the intensities of the gradient images in $\mathrm{x}$ and $\mathrm{y}$ direction; respectively. $\mathrm{E}$ is the edge strength and $\theta$ is the edge direction with the $\mathrm{x}$-axis.

Finally, the edges image is formed by the values of edges strength (E), and executed by the nonmaximum suppression algorithm and flux equilibrium check [28], to suppress thick edges to one pixel width and fill the missing pixels in the edge direction. Consequently, the final edges image, given in figure (6) is produced, which separates the patterns from surrounding noise. However, the edges do not provide information about where exactly are the inner of the patterns and where are their surroundings. 
Signal \& Image Processing : An International Journal (SIPIJ) Vol.3, No.2, April 2012

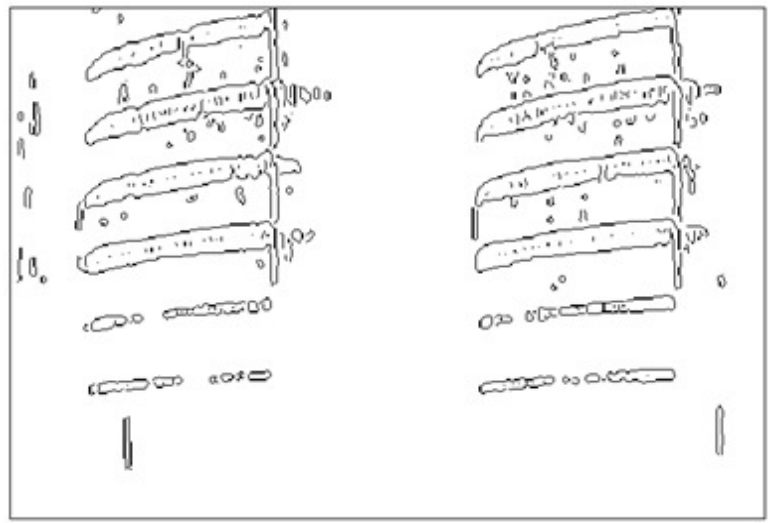

Figure 6. The edges image of the limited dynamic range spectrogram using $5 * 5$ mask and a flux check matrix of size $3 * 3$

\subsection{Reconstructing the spectrogram}

A classification condition is applied to each row and afterwards each column of the edges image. This condition compares the average power spectrum of all pixels among two subsequent edges in one row (column), with respect to the average power spectrum of these two edges, as clarified in the algorithm given in figure (7).

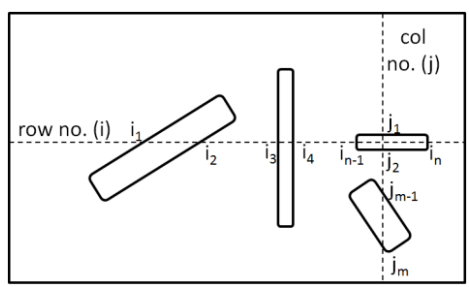

$$
\begin{aligned}
& I=\left\{i_{1}, i_{2}, \ldots, i_{n}\right\}, J=\left\{j_{1}, j_{2}, \ldots, j_{m}\right\} \\
& \text { for } k=1: n-1 \\
& \text { Pixels }\left(i_{k}: i_{k+1}\right)= \begin{cases}\text { sound pattern } \quad ; \operatorname{mean}\left(\operatorname{Power}\left(i_{k}: i_{k+1}\right)\right) \geq \operatorname{mean}\left(\operatorname{Power}\left(i_{k}, i_{k+1}\right)\right) \\
\text { surrounding noise } & ; \text { mean }\left(\operatorname{Power}\left(i_{k}: i_{k+1}\right)\right)<\operatorname{mean}\left(\operatorname{Power}\left(i_{k}, i_{k+1}\right)\right)\end{cases} \\
& \text { end } \\
& \text { for } k=1: m-1 \\
& \operatorname{Pixels}\left(j_{k}: j_{k+1}\right)= \begin{cases}\text { sound pattern } \quad ; \operatorname{mean}\left(\operatorname{Power}\left(j_{k}: j_{k+1}\right)\right) \geq \operatorname{mean}\left(\operatorname{Power}\left(j_{k}, j_{k+1}\right)\right) \\
\operatorname{surrounding} \text { noise } & ; \operatorname{mean}\left(\operatorname{Power}\left(j_{k}: j_{k+1}\right)\right)<\operatorname{mean}\left(\operatorname{Power}\left(j_{k}, j_{k+1}\right)\right)\end{cases} \\
& \text { end }
\end{aligned}
$$

Figure 7. Schematic diagram and algorithm for the classification condition which classifies sound patterns from their surrounding noise.

Thus, the patterns are defined and their power values are restored, and the surrounding noises are also defined and their power values are eliminated, results in the enhanced spectrogram shown in figure (8).

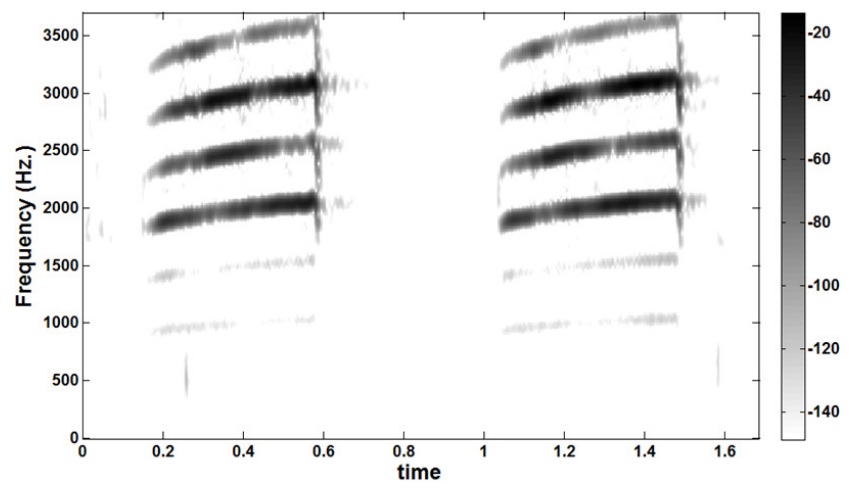

Figure 8 . The enhanced spectrogram by the proposed method revealing sound patterns and eliminating their surrounding noise. 


\section{Experimental results and discussion}

The results obtained by the proposed method [PM] were investigated and compared to those obtained by conventional and modern spectrogram enhancement methods. The designing parameters of these methods were carefully selected to give best enhanced spectrogram for the first application (Rhinolophus blasii bat), and were fixed over the following applications to have leading results and trustful comparisons. The first method is the widely used band pass filter [BP] with a band width enclosing the sound patterns, and measured at half-power points (i.e., gain -3 $\mathrm{dB}$ relative to peak). The second method is multi-band spectral subtraction [MBSS] using 4 linearly-spaced frequency bands, over subtraction factor of 4 and power factor of 1.5 [7]. The third method employs the Wiener filter [WF] with a spectral distance threshold of 3 and the initial 0.03 seconds considered as noise[11]. The fourth method is wavelet packet decomposition [WPD] with soft thresholding and 5 level decompositions using symlet 8 wavelet $[14,16]$. The number of tested sound samples are 42 (each with 23-25 sec for Rhinolophus blasii bat), 37 (each with 23-25 sec for Barbastella barbastellus bat), 48 (each with 0.55-0.9 sec for Vanellus vanellus bird), and 45 (each with 0.55-0.9 sec for Parus major bird), with a frame length of $0.025 \mathrm{sec}$ multiplied by Bartlett window function, and $90 \%$ overlapping percentage.

The analysis was applied by both subjective and objective measures of enhancement accuracy. The subjective measure is borrowed from the field of psychology and the human judgment of evaluation. One of the commonly used subjective measures is the Mean Opinion Score (MOS), which gives a numerical estimation of the perceived quality of the media received [30]. After enhancing the spectrogram, its time domain signal was reconstructed and played back to 10 listeners. These listeners ( 5 females, and 5 males) were asked to give a score $[1=\mathrm{bad}, 2=$ poor, 3 $=$ fair, $4=$ good, and $5=$ excellent $]$ to estimate the enhanced spectrogram quality. Afterwards, the MOS was calculated by averaging the given scores, and its confidence interval (CI) was computed for $95 \%$ confidence level, as described in figure (9).

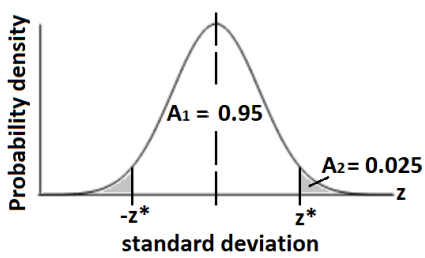

Figure 9. Normal distribution curve with $95 \%$ confidence level. $A_{2}=P\left(z>z^{*}\right)=(1-0.95) / 2$ $=0.025, \mathrm{P}\left(\mathrm{z}<=\mathrm{z}^{*}\right)=1-0.025=0.975$, results in $\mathrm{z}^{*}=1.96$ (from normal distribution table).

The confidence interval is(MOS $-[1.96 * \sigma]$, MOS $+[1.96 * \sigma])$. Where $\left[-z^{*}, z^{*}\right]$ encloses CI on the standard deviation axis (z) and $\sigma$ is the standard deviation of the opinion score.

On the other hand, objective measures are borrowed from digital signal processing and information theory, providing equations that can be used to measure the enhancement accuracy of the enhanced spectrogram in comparison to the clean one. Four widely used and easy to implement objective measures were employed, having high correlation with diagnostic acceptability [11,30,31]. These measures are the overall Signal to Noise Ratio (SNR), Segmental Signal to Noise Ratio (SSNR), Log Spectral Distance (LSD), and Itakura Saito (IS), given by equations $(3,8,9$, and 10; respectively).SSNR is defined as the average of SNR values over segments with sound activity, LSD is the spectral distance or distortion measure, expressed in $\mathrm{dB}$, between the enhanced and clean spectrograms, while IS a measure of the perceptual difference between these two spectrograms. Furthermore, the average eccentricity (AE) was calculated by equation (11), to simply check if the shape of sound patterns in the enhanced spectrogram was changed from those in the original spectrogram, results in indication of whether the enhanced spectrogram is augmented for further pattern recognition task. Eccentricity is the aspect ratio of length to width of the minimum rectangle bounding the sound pattern. 
Signal \& Image Processing : An International Journal (SIPIJ) Vol.3, No.2, April 2012

$$
\begin{aligned}
& S S N R=\frac{10}{M} \sum_{m=0}^{M-1} \log _{10} \sum_{i=N m}^{N m+N-1}\left(\frac{\sum P_{\text {clean }}}{\sum\left|P_{\text {clean }}-P_{\text {output }}\right|}\right) \\
& L S D=\sqrt{\frac{2}{F S} \sum_{0}^{F S / 2}\left[10 \log _{10}\left(\frac{P_{\text {clean }}}{P_{\text {output }}}\right)\right]^{2}} \\
& I S=\frac{2}{F S} \sum_{0}^{F S / 2}\left[\frac{P_{\text {clean }}}{P_{\text {output }}}-\log _{10} \frac{P_{\text {clean }}}{P_{\text {output }}}-1\right] \\
& A E=\frac{1}{K} \sum_{j=1}^{K} \frac{L_{j}}{W_{j}}
\end{aligned}
$$

Where FS is the sampling rate of the signal, $\mathrm{P}_{\text {clean }}$ and $\mathrm{P}_{\text {output }}$ are the clean and enhanced power spectrum respectively. $\mathrm{M}$ is the number of spectrogram segments (set to 20), $\mathrm{N}$ is the number of samples on a segment. $\mathrm{L}$ and $\mathrm{W}$ are the length and width; respectively, for the minimum rectangle bounding the sound pattern, and $\mathrm{K}$ is the number of sound patterns in the spectrogram.

The applications were selected to cover different spectrogram shapes, including those with narrow band, wide band, constant frequency, frequency modulated, short pulses, and long pulses patterns. The original bioacoustics calls in each application were corrupted by several white Gaussian noises, as descriptively shown in the left part of figure (10) for a saw tooth wave, result in several time domain SNRs $(30,20,10,5$, and $1 \mathrm{~dB})$, and in correspondence several spectrogram SNRs as demonstrated in the right part of figure (10), with average values of (3.66, $2.89,2,1.73,1.52 \mathrm{~dB}$; respectively).For each application and for each SNR, the five enhancement methods (BP, MBSS, WF, WPD, and PM) were applied, and the results were subjectively and objectively compared. It is worthy to mention that the silent regions were being removed, because they can considerably influence the output objective measures.
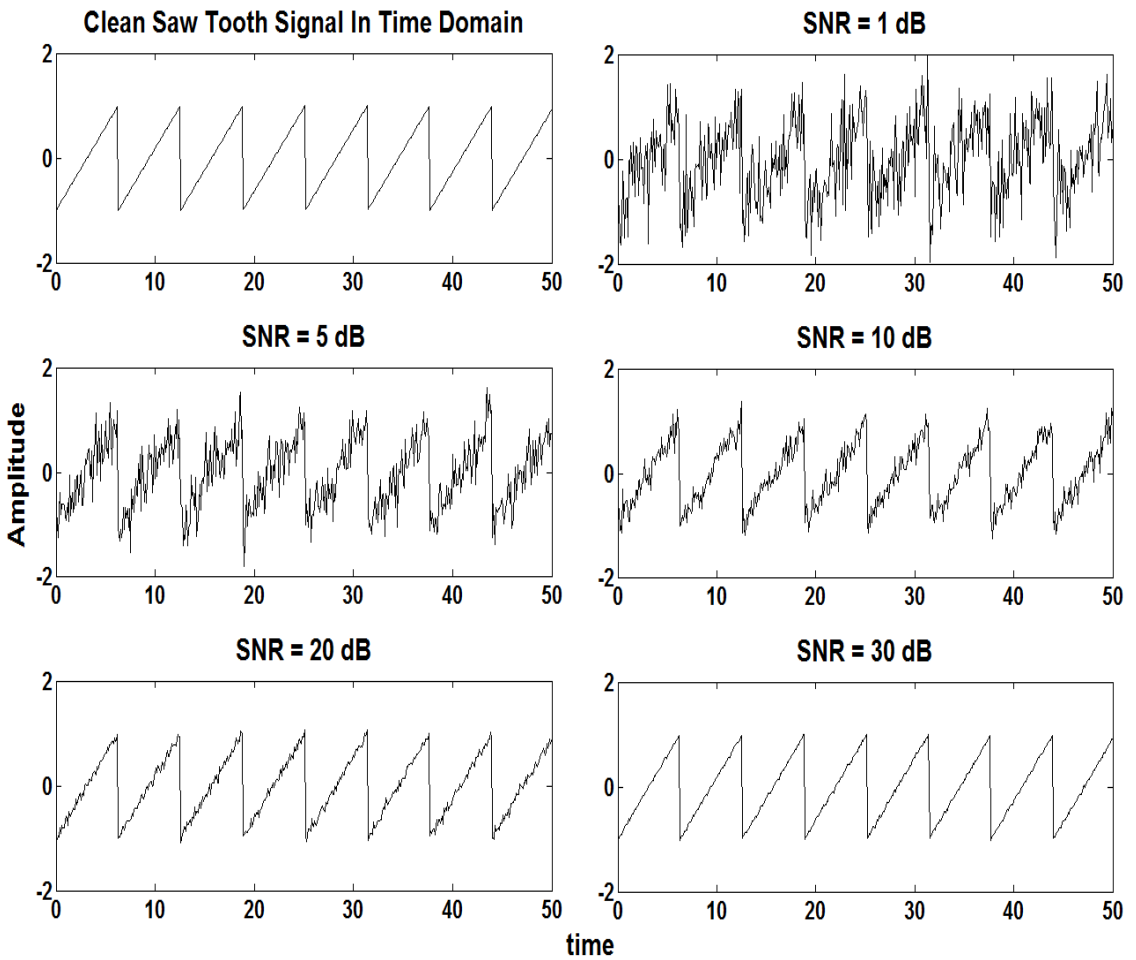
Signal \& Image Processing : An International Journal (SIPIJ) Vol.3, No.2, April 2012

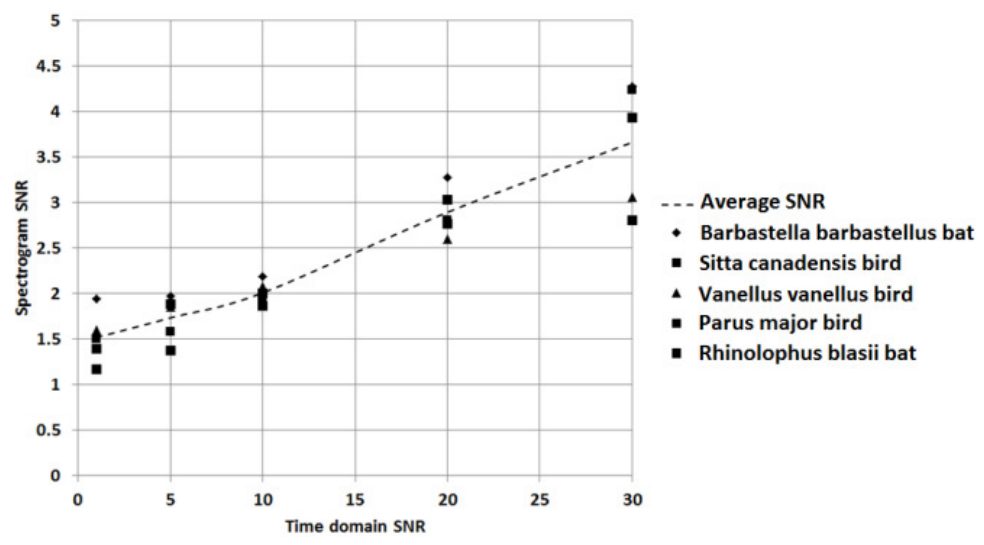

Figure 10. (top) A description for how the addition of different white Gaussian noise changes the structure and SNR of the time domain signal. (bottom) The corresponding changes in the spectrogram SNR averaged for the five applications.

\subsection{Bioacoustics call of Rhinolophus blasii bat}

The echolocation call of the Rhinolophus blasii bat which was investigated in this section includes medium duration strong sound pulses separated by short intervals. These pulses cover short Frequency Modulated (FM) band around $5 \mathrm{KHz}$, roughly estimated as Constant Frequency (CF) band, which were slowed down by a time expansion factor of 10 to be in the audible range, as shown in figure (11a).BP approach was able to remove most of the added noise, by rejecting the spectrogram values outside the small band surrounds the frequency of $5 \mathrm{KHz}$, as graphically shown in figure (11b) and numerically in the second column(s) of Table 1. Instead, the MBSS and WF approaches were not successful to remove reasonable amount of noise, especially for input SNRs less than $20 \mathrm{~dB}$. The spectrograms generated with MBSS approach tended to temporally spread out the sound pulses, while those generated by WF approach tended to temporally cut from the duration of the pulses, as displayed in figure (11c, 11d) and the third and fourth column(s) of Table 1; respectively.

(a)

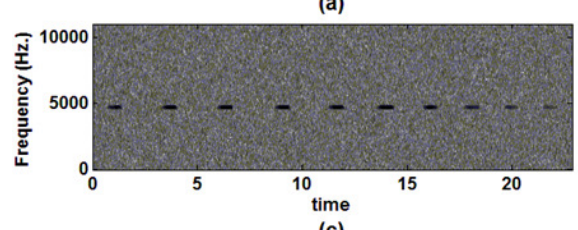

(c)

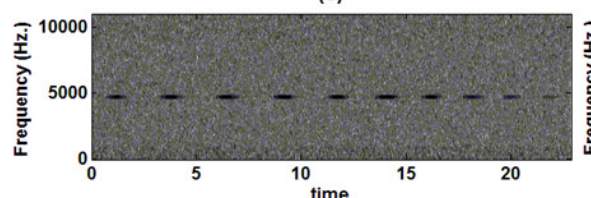

(e)

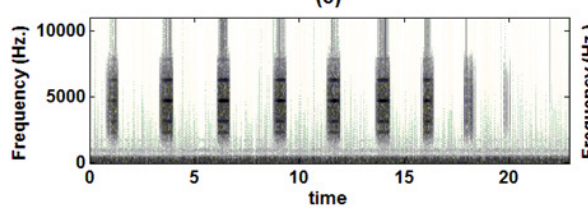

(b)

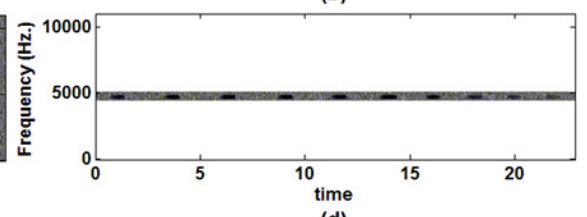

(d)

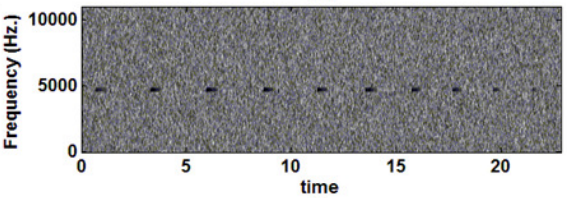

(f)

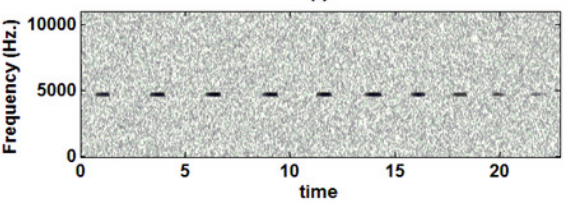

Figure 11. (a) One of Rhinolophus Blasii bat echolocation calls at SNR $=1 \mathrm{~dB}$ (with expansion factor of 10), and its enhanced spectrogram by (b) BP, (c) MBSS, (d) WF, (e) WPD, and (f) PM. 
Signal \& Image Processing : An International Journal (SIPIJ) Vol.3, No.2, April 2012

For higher values of input SNRs, the WF presented better enhancement. The WPD approach removed reasonable amount of noise and presented good estimation for the time domain resolution of the sound pulses. However, the five level decompositions produced repeated patterns along the frequency axis, as shown in figure (11e) and the fifth column(s) of Table 1. The PM dealt with the noisy spectrogram as an image, and was able to preserve the sound pulses while removing most of the attached noise, as expressed in figure (11f) and the sixth column(s) of Table 1.

Table 1. The subjective and objective measures for the spectrogram of Rhinolophus Blasii call enhanced by BP, MBSS, WF, WPD, and PM

(A) MOS

\begin{tabular}{|c|c|c|c|c|c|c|}
\hline $\begin{array}{c}\text { input } \\
\text { SNR }\end{array}$ & noisy & BP & MBSS & WF & WPD & PM \\
\hline 1 & $1(1,1)$ & $2.8(2.5,3)$ & $1(1,1)$ & $1.1(0.9,1.3)$ & $1.7(1.4,2)$ & $1.6(1.3,1.9)$ \\
\hline 5 & $1.1(0.9,1.3)$ & $2.9(2.7,3.1)$ & $1(1,1)$ & $1.2(0.9,1.5)$ & $1.8(1.5,2)$ & $2(1.6,2.4)$ \\
\hline 10 & $1.3(1,1.6)$ & $2.8(2.5,3)$ & $1.1(0.9,1.3)$ & $1.3(1,1.6)$ & $1.8(1.4,2.2)$ & $2.6(2.3,2.9)$ \\
\hline 20 & $1.3(0.9,1.7)$ & $3.1(2.9,3.3)$ & $1.3(0.9,1.7)$ & $1.8(1.3,2.3)$ & $1.9(1.5,2.2)$ & $4.9(4.7,5.1)$ \\
\hline 30 & $1.6(1.3,1.9)$ & $3.3(3,3.6)$ & $1.5(1.1,1.9)$ & $2.5(2.2,2.8)$ & $2(1.7,2.3)$ & $5(5,5)$ \\
\hline
\end{tabular}

(B) SNR

\begin{tabular}{|c|c|c|c|c|c|c|}
\hline input SNR & noisy & BP & MBSS & WF & WPD & PM \\
\hline 1 & 2.238 & 15.399 & 2.1390 & 2.565 & 7.734 & 6.903 \\
\hline 5 & 2.579 & 15.711 & 2.505 & 3.227 & 8.695 & 8.936 \\
\hline 10 & 3.097 & 16.207 & 3.017 & 3.983 & 8.912 & 13.594 \\
\hline 20 & 4.344 & 17.322 & 4.200 & 7.786 & 9.319 & 31.798 \\
\hline 30 & 5.982 & 18.700 & 5.696 & 13.142 & 10.113 & 31.930 \\
\hline
\end{tabular}

\begin{tabular}{|c|c|c|c|c|c|c|}
\hline input SNR & noisy & BP & MBSS & WF & WPD & PM \\
\hline 1 & 1.755 & 3.278 & 1.744 & 1.793 & 2.472 & 2.292 \\
\hline 5 & 1.794 & 3.312 & 1.786 & 1.869 & 2.667 & 2.526 \\
\hline 10 & 1.854 & 3.369 & 1.845 & 1.956 & 2.765 & 3.063 \\
\hline 20 & 1.998 & 3.495 & 1.981 & 2.395 & 2.859 & 6.470 \\
\hline 30 & 2.187 & 3.651 & 2.155 & 3.057 & 3.004 & 5.187 \\
\hline
\end{tabular}

(D) LSD

\begin{tabular}{|c|c|c|c|c|c|c|}
\hline input SNR & noisy & BP & MBSS & WF & WPD & PM \\
\hline 1 & 1.260 & 0.537 & 1.834 & 2.125 & 1.011 & 0.767 \\
\hline 5 & 1.002 & 0.352 & 1.826 & 2.020 & 0.655 & 0.620 \\
\hline 10 & 1.050 & 0.501 & 1.708 & 1.743 & 0.520 & 0.560 \\
\hline 20 & 0.697 & 0.312 & 1.500 & 1.267 & 0.218 & 0.311 \\
\hline 30 & 0.528 & 0.279 & 1.256 & 1.044 & 0.144 & 0.279 \\
\hline
\end{tabular}

(E) IS

\begin{tabular}{|c|c|c|c|c|c|c|}
\hline input SNR & noisy & BP & MBSS & WF & WPD & PM \\
\hline 1 & 1.281 & 0.186 & 3.455 & 5.296 & 0.833 & 0.392 \\
\hline 5 & 0.731 & 0.076 & 3.410 & 4.559 & 0.334 & 0.242 \\
\hline 10 & 0.815 & 0.155 & 2.828 & 2.997 & 0.216 & 0.191 \\
\hline 20 & 0.313 & 0.056 & 1.994 & 1.294 & 0.049 & 0.054 \\
\hline 30 & 0.169 & 0.043 & 1.266 & 0.803 & 0.023 & 0.043 \\
\hline
\end{tabular}

(F) AE

\begin{tabular}{|c|c|c|c|c|c|c|}
\hline input SNR & noisy & BP & MBSS & WF & WPD & PM \\
\hline 1 & 3.51 & 3.51 & 4.21 & 2.75 & 5.16 & 3.52 \\
\hline 5 & 3.51 & 3.51 & 4.21 & 2.79 & 5.15 & 3.52 \\
\hline 10 & 3.51 & 3.51 & 3.97 & 3.06 & 4.99 & 3.52 \\
\hline 20 & 3.51 & 3.51 & 3.84 & 3.11 & 4.64 & 3.52 \\
\hline 30 & 3.51 & 3.51 & 3.75 & 3.14 & 4.32 & 3.52 \\
\hline
\end{tabular}


Signal \& Image Processing : An International Journal (SIPIJ) Vol.3, No.2, April 2012

\subsection{Bioacoustics call of Barbastellabarbastellus bat}

As an alternative, the echolocation call of the Barbastella barbastellus bat includes very short duration sound pulses separated by non-fixed intervals. The pulses cover long Frequency Modulated (FM) band over the region [25-100] KHz, which were also slowed down by a time expansion factor of 10 to be in the audible range, as demonstrated in figure (12a). Since the frequency band covered by the sound pulses is large, BP approach was not able to remove most of the noise into this band, as graphically shown in figure (12b) and numerically in the second column(s) of Table 2. Meanwhile, the pulses were temporally spread out by MBSS approach; however, it removed reasonable amount of noise, as displayed in figure (12c) and the third column(s) of Table 2. The WF approach removed higher amount of noise except those exist in the beginning of the signal. There are also spectral cut from the pulses along the frequency axis and the temporal resolution is degraded, as given in figure (12d) and the fourth column(s) of Table 2. Although the spectral resolution of the spectrograms generated by the WPD approach still corrupt and low frequency noise was not removed, there were no possibility for the repetition of the pulses along the frequency axis because the covered FM band is considerably high, as clarified in figure (12e) and the fifth column(s) of Table 2. The PM has the same performance as in application 1, by keeping the temporal and spectral properties of the pulses while removed most of the attached noise, as shown in figure (12f) and the sixth column(s) of Table 2.

(a)

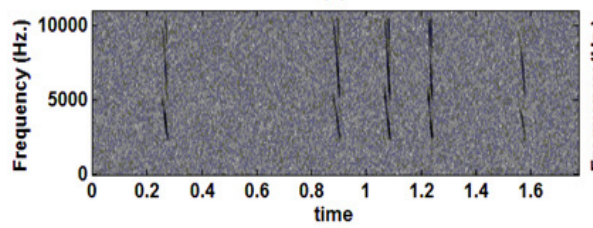

(c)

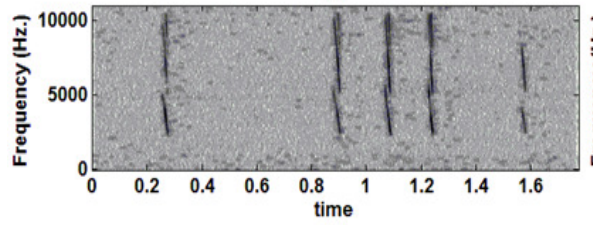

(e)

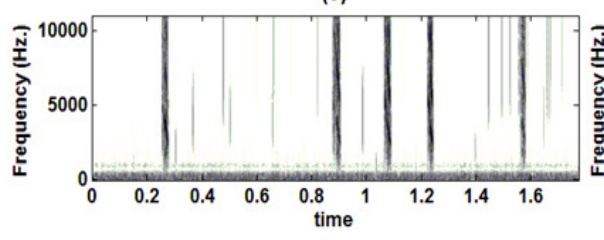

(b)

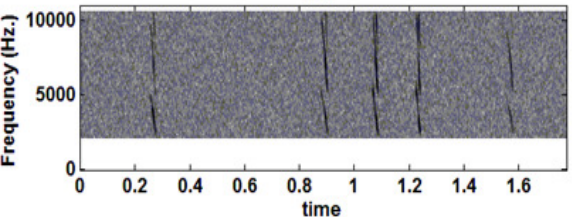

(d)

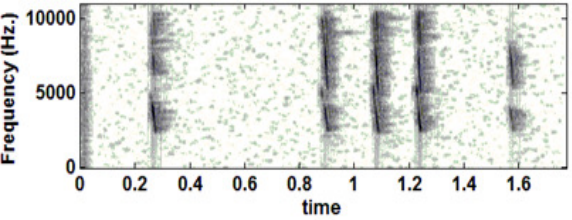

(f)

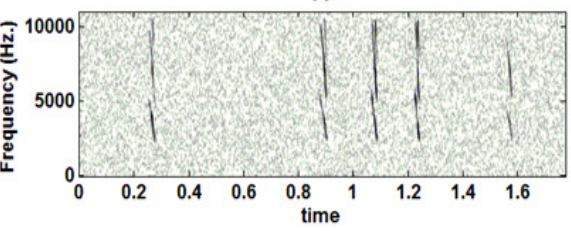

Figure 12. (a) One of Barbastella Barbastellus bat echolocation calls at $\mathrm{SNR}=5 \mathrm{~dB}$ (with expansion factor of 10), and its enhanced spectrogram by (b) BP, (c) MBSS, (d) WF, (e) WPD, and (f) PM. 
Signal \& Image Processing : An International Journal (SIPIJ) Vol.3, No.2, April 2012

Table 2. The subjective and objective measures for the spectrogram of Barbastella barbastellus call enhanced by BP, MBSS, WF, WPD, and PM

(A) MOS

\begin{tabular}{|c|c|c|c|c|c|c|}
\hline $\begin{array}{c}\text { input } \\
\text { SNR }\end{array}$ & noisy & BP & MBSS & WF & WPD & PM \\
\hline 1 & $1(1,1)$ & $1.2(0.9,1.5)$ & $1.3(1,1.6)$ & $2.1(1.9,2.3)$ & $2.7(2.4,3)$ & $2(1.7,2.3)$ \\
\hline 5 & $1.1(0.9,1.3)$ & $1.3(1,1.6)$ & $1.4(1.1,1.7)$ & $2.4(2.1,2.7)$ & $2.8(2.5,3.1)$ & $2.4(2.1,2.7)$ \\
\hline 10 & $1.3(0.9,1.7)$ & $1.4(1,1.8)$ & $1.5(1.2,1.8)$ & $2.6(2.3,2.9)$ & $2.9(2.7,3.1)$ & $3.4(3.1,3.7)$ \\
\hline 20 & $1.4(1,1.7)$ & $1.6(1.3,1.9)$ & $1.9(1.7,2.1)$ & $2.8(2.4,3.2)$ & $3.1(2.9,3.3)$ & $5(5,5)$ \\
\hline 30 & $1.7(1.4,2)$ & $1.9(1.7,2.1)$ & $2.3(2,2.6)$ & $2.9(2.7,3.1)$ & $3.2(2.9,3.5)$ & $5(5,5)$ \\
\hline
\end{tabular}

(B) SNR

\begin{tabular}{|c|c|c|c|c|c|c|}
\hline input SNR & noisy & BP & MBSS & WF & WPD & PM \\
\hline 1 & 2.303 & 3.379 & 3.757 & 7.918 & 10.939 & 7.140 \\
\hline 5 & 2.662 & 3.760 & 4.307 & 9.272 & 11.595 & 9.500 \\
\hline 10 & 3.181 & 4.273 & 5.023 & 10.456 & 12.149 & 14.289 \\
\hline 20 & 4.393 & 5.454 & 6.937 & 11.691 & 13.089 & 22.826 \\
\hline 30 & 5.745 & 6.732 & 9.236 & 11.917 & 13.182 & 22.872 \\
\hline
\end{tabular}

(C) SSNR

\begin{tabular}{|c|c|c|c|c|c|c|}
\hline input SNR & noisy & BP & MBSS & WF & WPD & PM \\
\hline 1 & 1.763 & 1.886 & 1.931 & 2.510 & 2.843 & 2.320 \\
\hline 5 & 1.804 & 1.930 & 1.995 & 2.782 & 2.961 & 2.592 \\
\hline 10 & 1.864 & 1.989 & 2.078 & 3.182 & 3.077 & 3.158 \\
\hline 20 & 2.004 & 2.126 & 2.312 & 5.017 & 3.376 & 6.814 \\
\hline 30 & 2.163 & 2.278 & 2.659 & 4.233 & 3.628 & 3.386 \\
\hline
\end{tabular}

(D) LSD

\begin{tabular}{|c|c|c|c|c|c|c|}
\hline input SNR & noisy & BP & MBSS & WF & WPD & PM \\
\hline 1 & 0.942 & 1.015 & 0.600 & 0.194 & 0.278 & 0.610 \\
\hline 5 & 0.943 & 0.888 & 0.466 & 0.140 & 0.191 & 0.485 \\
\hline 10 & 0.859 & 0.715 & 0.417 & 0.209 & 0.171 & 0.360 \\
\hline 20 & 0.571 & 0.535 & 0.244 & 0.201 & 0.124 & 0.121 \\
\hline 30 & 0.407 & 0.367 & 0.135 & 0.392 & 0.173 & 0.195 \\
\hline
\end{tabular}

(E) IS

\begin{tabular}{|c|c|c|c|c|c|c|}
\hline input SNR & noisy & BP & MBSS & WF & WPD & PM \\
\hline 1 & 0.630 & 0.777 & 0.228 & 0.036 & 0.059 & 0.234 \\
\hline 5 & 0.631 & 0.564 & 0.133 & 0.021 & 0.032 & 0.141 \\
\hline 10 & 0.506 & 0.341 & 0.107 & 0.025 & 0.020 & 0.074 \\
\hline 20 & 0.203 & 0.179 & 0.042 & 0.024 & 0.015 & 0.008 \\
\hline 30 & 0.100 & 0.082 & 0.022 & 0.071 & 0.018 & 0.021 \\
\hline
\end{tabular}

(F) AE

\begin{tabular}{|c|c|c|c|c|c|c|}
\hline input SNR & noisy & BP & MBSS & WF & WPD & PM \\
\hline 1 & 57 & 57 & 56.6 & 6 & 16.27 & 57 \\
\hline 5 & 57 & 57 & 56.8 & 9.31 & 19 & 57 \\
\hline 10 & 57 & 57 & 56.8 & 14.54 & 34.52 & 57 \\
\hline 20 & 57 & 57 & 56.85 & 21.13 & 47.33 & 57 \\
\hline 30 & 57 & 57 & 56.85 & 30.46 & 54.18 & 57 \\
\hline
\end{tabular}

\subsection{Bioacoustics call of Vanellus vanellus bird}

As an example for a multi harmonic sound stream in the human hearing range, the bioacoustics call of Vanellus vanellus bird was investigated in the region bounded by $6 \mathrm{KHz}$. The sound stream contains three FM long pulses with dominant frequencies around $(1,2.2$, and 4$) \mathrm{KHz}$; respectively, followed by three downstream $\mathrm{CF}$ short pulses of fundamental frequencies around $(2,3$, and 4$) \mathrm{KHz}$; respectively, as given in figure (13a). Enhancement by the BP approach did not produce clear spectrogram, since the pulses cover much of the frequency axis, given high 
constraint to the rejected band by this approach, as shown in figure (13b) and the second column(s) of Table 3. The enhanced spectrogram generated by the MBSS approach has reasonable temporal resolution and degraded spectral resolution of the sound pulses, especially for the downstream pulses which corrupted by high spectral distortion, as displayed in figure (13c) and the third column(s) of Table 3.On the other hand, the temporal and spectral resolutions of the spectrogram generated by WF approach are acceptable, although there is little spectral leakage for the downstream pulses and initial sound noise $(<0.03 \mathrm{sec})$ were not removed, as plotted in figure (13d) and the fourth column(s) of Table 3. Whereas the low frequency noise was not enhanced by the WPD approach, many temporal bands of noises were removed. The decomposition of the sound stream presented spectral mirrors of the weak harmonic patterns and almost eliminated the downstream pulses, as demonstrated in figure (13e) and the fifth column(s) of Table 3. Meanwhile, the enhanced spectrogram by PMre established high temporal and spectral resolutions of the sound pulses, as indicated by the obtained LSD, with high distinction from the attached noise, as designated by the obtained SNR and shown in figure (13f) and the sixth column(s) of Table 3.
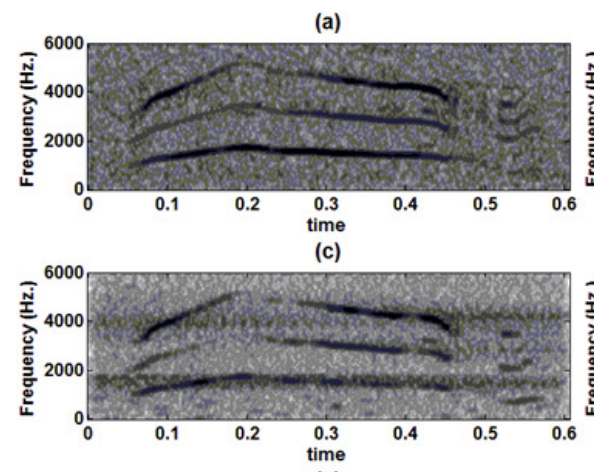

(e)

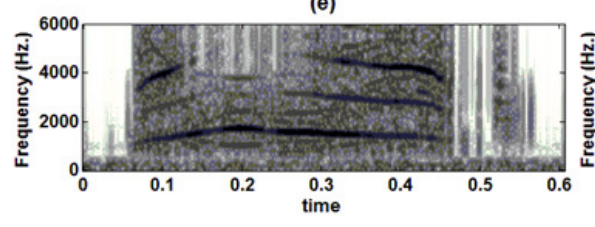

(b)

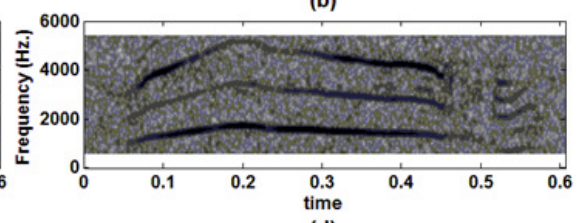

(d)

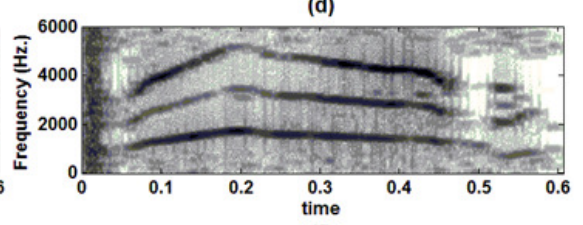

(f)

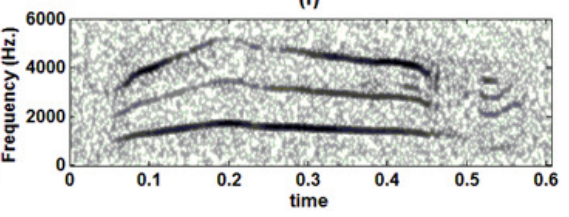

Figure 13. (a) One of Vanellus vanellus bird calls at $\mathrm{SNR}=10 \mathrm{~dB}$, and its enhanced spectrogram by (b) BP, (c) MBSS, (d) WF, (e) WPD, and (f) PM.

Table 3. The subjective and objective measures for the spectrogram of Vanellus vanellus bird call enhanced by BP, MBSS, WF, WPD, and PM

(A) MOS

\begin{tabular}{|c|c|c|c|c|c|c|}
\hline $\begin{array}{c}\text { input } \\
\text { SNR }\end{array}$ & noisy & BP & MBSS & WF & WPD & PM \\
\hline 1 & $1(1,1)$ & $1.5(1.2,1.8)$ & $1.4(1.1,1.7)$ & $2(1.7,2.3)$ & $1.7(1.4,2)$ & $2.4(2.1,2.7)$ \\
\hline 5 & $1.4(1.1,1.7)$ & $1.6(1.3,1.9)$ & $1.5(1.2,1.8)$ & $2.2(1.9,2.5)$ & $1.8(1.4,2.2)$ & $2.9(2.7,3.1)$ \\
\hline 10 & $1.3(1,1.6)$ & $1.8(1.4,2.2)$ & $1.7(1.3,2.1)$ & $2.4(2.1,2.7)$ & $1.9(1.7,2.1)$ & $3.7(3.4,4)$ \\
\hline 20 & $1.5(1.2,1.8)$ & $1.9(1.5,2.3)$ & $1.8(1.4,2.2)$ & $2.6(2.3,2.9)$ & $1.9(1.7,2.1)$ & $4.7(4.4,5)$ \\
\hline 30 & $1.6(1.3,1.9)$ & $2(1.6,2.4)$ & $1.8(1.4,2.2)$ & $2.7(2.4,3)$ & $1.9(1.7,2.1)$ & $5(5,5)$ \\
\hline
\end{tabular}

(B) SNR

\begin{tabular}{|c|c|c|c|c|c|c|}
\hline input SNR & noisy & BP & MBSS & WF & WPD & PM \\
\hline 1 & 1.651 & 2.635 & 2.489 & 3.591 & 2.895 & 4.371 \\
\hline 5 & 1.941 & 2.843 & 2.689 & 4.046 & 3.180 & 5.414 \\
\hline 10 & 2.207 & 3.153 & 2.912 & 4.452 & 3.317 & 6.807 \\
\hline 20 & 2.662 & 3.532 & 3.108 & 4.771 & 3.422 & 9.078 \\
\hline 30 & 2.814 & 3.605 & 3.163 & 4.884 & 3.339 & 9.480 \\
\hline
\end{tabular}


Signal \& Image Processing : An International Journal (SIPIJ) Vol.3, No.2, April 2012

(C) SSNR

\begin{tabular}{|c|c|c|c|c|c|c|}
\hline input SNR & noisy & BP & MBSS & WF & WPD & PM \\
\hline 1 & 1.688 & 1.801 & 1.784 & 1.925 & 1.896 & 2.001 \\
\hline 5 & 1.721 & 1.825 & 1.807 & 1.995 & 1.989 & 2.121 \\
\hline 10 & 1.752 & 1.861 & 1.833 & 2.059 & 2.036 & 2.283 \\
\hline 20 & 1.804 & 1.904 & 1.856 & 2.119 & 2.120 & 2.564 \\
\hline 30 & 1.822 & 1.913 & 1.863 & 2.162 & 1.991 & 2.621 \\
\hline
\end{tabular}

(D) LSD

\begin{tabular}{|c|c|c|c|c|c|c|}
\hline input SNR & noisy & BP & MBSS & WF & WPD & PM \\
\hline 1 & 1.172 & 1.083 & 0.982 & 1.220 & 1.009 & 0.790 \\
\hline 5 & 1.042 & 0.938 & 0.876 & 1.141 & 0.907 & 0.505 \\
\hline 10 & 0.988 & 0.888 & 0.537 & 1.028 & 0.783 & 0.537 \\
\hline 20 & 0.814 & 0.783 & 0.753 & 1.012 & 0.587 & 0.267 \\
\hline 30 & 0.718 & 0.714 & 0.515 & 0.968 & 0.579 & 0.267 \\
\hline
\end{tabular}

(E) IS

\begin{tabular}{|c|c|c|c|c|c|c|}
\hline input SNR & noisy & BP & MBSS & WF & WPD & PM \\
\hline 1 & 0.630 & 0.922 & 0.703 & 1.221 & 0.856 & 0.423 \\
\hline 5 & 0.803 & 0.649 & 0.538 & 1.031 & 0.657 & 0.156 \\
\hline 10 & 0.707 & 0.568 & 0.182 & 0.804 & 0.469 & 0.177 \\
\hline 20 & 0.451 & 0.425 & 0.385 & 0.771 & 0.248 & 0.041 \\
\hline 30 & 0.341 & 0.345 & 0.169 & 0.696 & 0.233 & 0.041 \\
\hline
\end{tabular}

(F) $\mathrm{AE}$

\begin{tabular}{|c|c|c|c|c|c|c|}
\hline input SNR & noisy & BP & MBSS & WF & WPD & PM \\
\hline 1 & 8.47 & 8.47 & 14.63 & 10.32 & 3.13 & 8.51 \\
\hline 5 & 8.47 & 8.47 & 12.51 & 10.3 & 3.82 & 8.51 \\
\hline 10 & 8.47 & 8.47 & 9.78 & 9.46 & 4.17 & 8.51 \\
\hline 20 & 8.47 & 8.47 & 9.12 & 8.72 & 7.12 & 8.51 \\
\hline 30 & 8.47 & 8.47 & 8.65 & 8.19 & 7.44 & 8.51 \\
\hline
\end{tabular}

From the pattern recognition point of view, different $\mathrm{AE}$ values to those of the original spectrogram, reflect changing in the shape of some or all of the sound patterns, which result in non-accuracy in further pattern recognition results. Unlike, similar AE values do not ensure the shape of the sound pattern is similar to its original shape, but it may changes in a way that its aspect ratio is constant.

\section{Extended applications}

The spectrograms obtained by the proposed method (PM) displayed how it is powerful and consistent to enhance different structure bioacoustics calls. Therefore, these enhanced spectrograms can be implemented in various post processing tasks. In this section, the three most important implementations of the generated spectrogram by PM will be explored.

\subsection{Power and frequency contours}

The three variables of the enhanced spectrogram (i.e., time, frequency, and power spectrum) may be plotted in different orders to obtain its power and/or frequency contours, as visible for the bioacoustics call of Rhinolophus hipposideros bat (with expansion factor of 13) in figure (14), after its spectrogram was enhanced by PM. The power contours provides an image of the instantaneous power contents of the sound patterns and can be used for specific sound power detection after calculating the areas enclosed by these contours. While the frequency contours provide an image of the instantaneous frequency contents of the recorded bioacoustics call and can be used for designing more reliable frequency filters. 

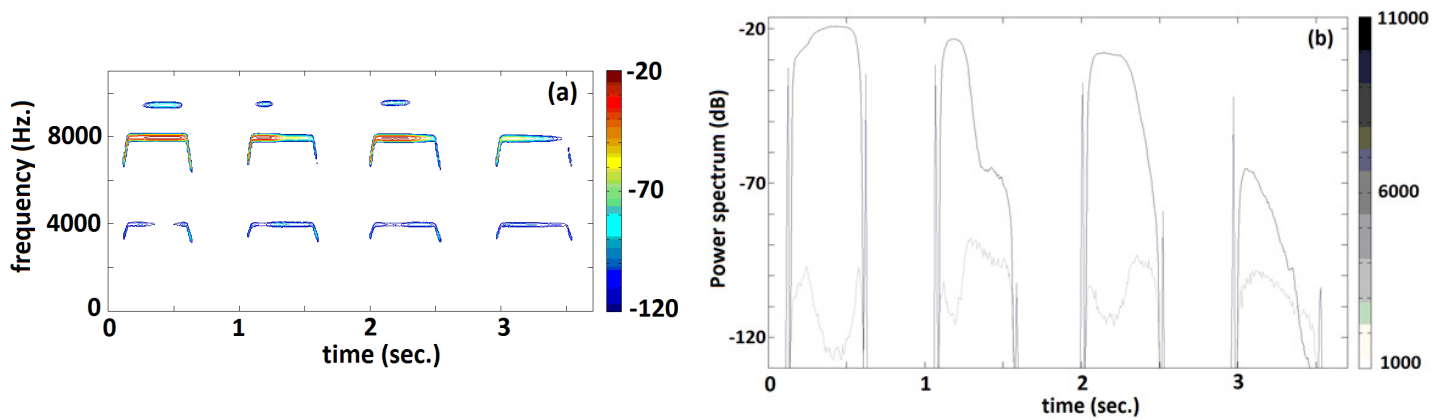

Figure 14. The enhanced (a) power and (b) frequency contours for Rhinolophus hipposideros bat call

\subsection{The enhanced wave form of the bioacoustics call}

By transforming the enhanced spectrogram variables back to the time domain by Inverse Fast Fourier Transform (IFFT), the enhanced wave form of the call is obtained, as given in figure (15). The phase information obtained through the former Short Time Fourier Transform (STFT) is used to reconstruct the enhanced wave form, following the flowchart of figure (15). The output waveform can be used for reliable extraction of the bioacoustics temporal features suchlike zero crossing rate, short time energy, temporal roll-off, and temporal spread of the sound patterns [1].
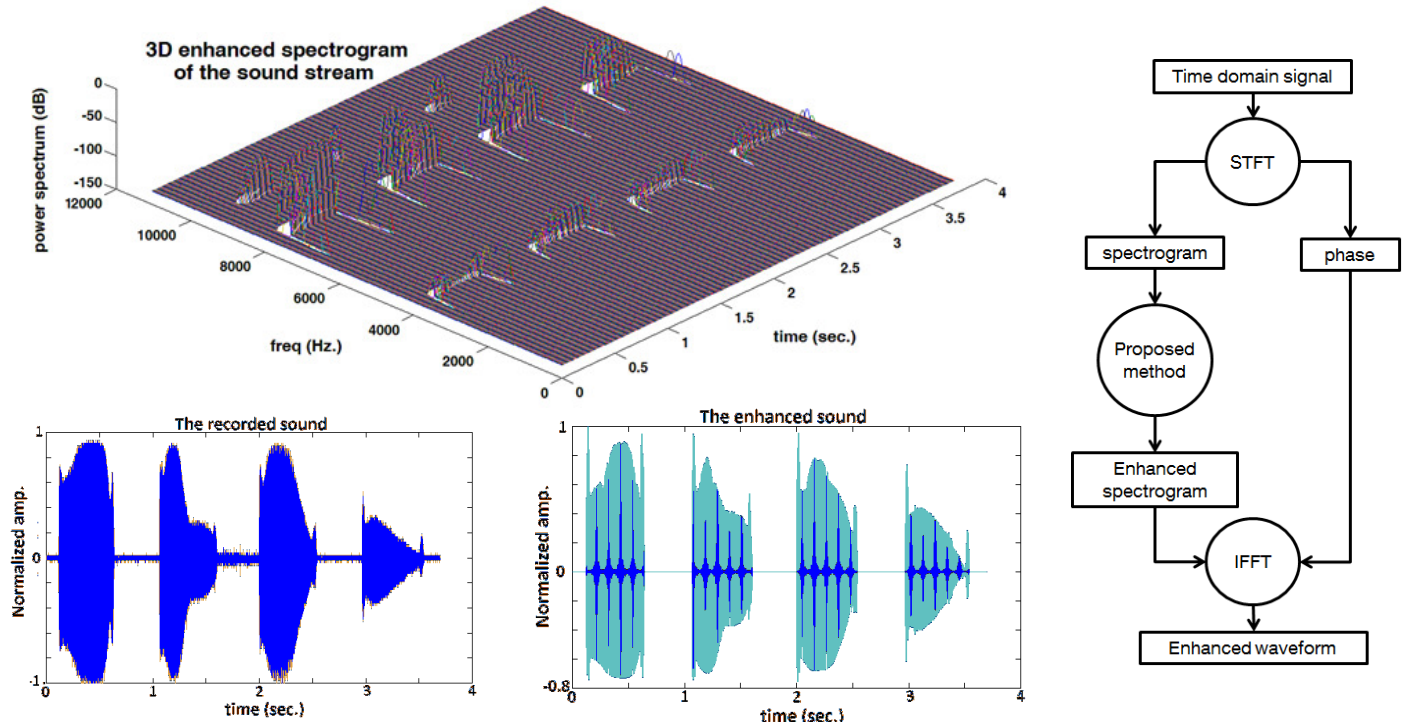

Figure 15. (upper) The 3D enhanced spectrogram for the bioacoustics call of Rhinolophus hipposideros bat(with expansion factor of 13)and flow chart to reconstruct the waveform of its sound stream. (bottom) the original and the reconstructed wave form of the sound stream; respectively.

\subsection{Bioacoustics calls classification}

From the enhanced spectrogram, simple and reduced number of 1D featuresand/or 2D features can be extracted for complete pattern recognition of the bioacoustics sound. The 1D features are the signal features suchlike the covered frequency band(s), peak frequency, pulse duration, interval between sound pulses, etc. while the 2D features are the image features suchlike eccentricity and centroid. As a test case, a classifier of Vanellus vanellus, Parus major, and Sitta 
Canadensis birds has been constructed using the eccentricity $(\mathrm{a} / \mathrm{b})$ and the vertical coordinate of the centroid (c) features, as shown in figure (16(ii)), extracted from the enhanced spectrogram of each bird sound, as displayed in figure (16(i)). The classifier was trained by 26, 30, and 37 sound patterns of the three birds; respectively, to define the rough dividing contours, given in figure (16(iii)). Afterwards, the classifier was tested by another 7, 8, and 10 sound patterns of the three birds; respectively, beside 7 patterns of Barbastella barbastellus bat and 5 patterns of Rhinolophus Blasii bat, giving $94.59 \%$ classification accuracy (two sound patterns of Sitta Canadensis wrongly detected as Vanellus vanellus), as plotted in figure (16(iii)). It may be realized that even simple classifier can separate out the sound patterns into the correct bioacoustics source, providing that distinctive features were selected and sufficient training patterns were used.

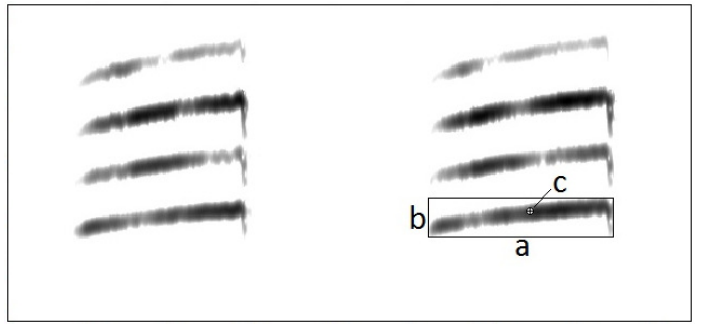

(i)

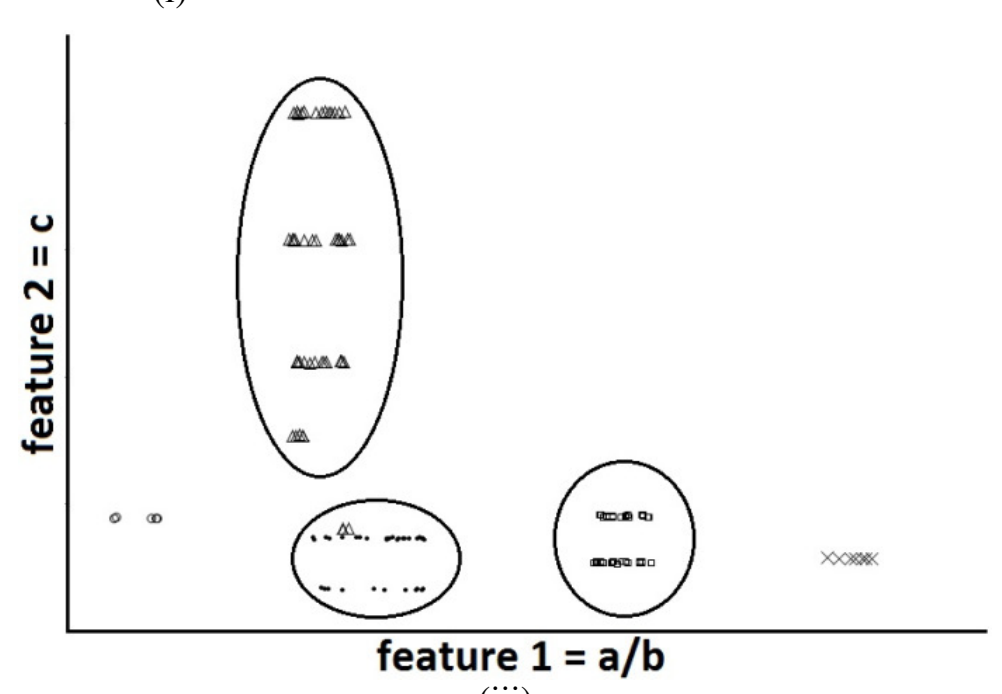

(iii)

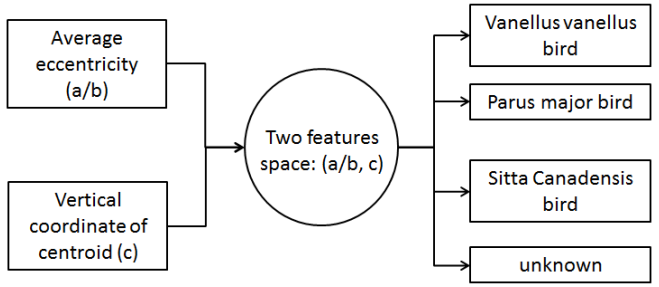

(ii)

Figure 16. (i) Enhanced spectrogram for one of Sitta Canadensis bird contains the strongestsound patterns, indicating how the eccentricity $(\mathrm{a} / \mathrm{b})$ and the vertical coordinate of the centroid (c) are extracted for one of its sound patterns. The units of $a$ and $b$ are in pixeld, and $\mathrm{c}$ in pixel number. (ii) Simple classifier structure with input of the two features, which form the classification space, and the outputs are four classes for Vanellus vanellus, Parus major, Sitta Canadensis , and unknown sounds. (iii) The classification space with three dividing contours encounters the features of the three birds, respectively, at which the surrounded region is for unknown sound features. The classification results also included with two sound patterns of Sitta Canadensis wrongly detected as Vanellus vanellus.

\section{Conclusion}

Spectrogram reading provides a direct method for hands-on learning of the characteristics of bioacoustics calls, therefore, a variety of enhancement techniques have been considered over the past years to remove the attached noises. In this paper, a spectrogram enhancement method was developed based on high accurate edge detection of the enclosed sound patterns and removing the 
Signal \& Image Processing : An International Journal (SIPIJ) Vol.3, No.2, April 2012

surrounding noise. The crest factor was presented as a smoothed version of the spectrogram image, avoiding the threshold problem of usual smoothing filters, suchlike Gaussian filter in Canny edge detector. The proposed method was applied to enhance the limited dynamic range spectrogram of different structure bioacoustics calls, in comparison to the four commonly used enhancement approaches, which are band pass filter (BP), multi-band spectral subtraction (MBSS), Wiener filter (WF), and wavelet packet decomposition (WPD) approaches. The comparison was established on one subjective measure [mean opinion score] and four objective measures [signal to noise ratio, segmental signal to noise ratio, log spectral distance, and Itakura Saito] of the spectrograms obtained by the five methods at different SNR. The results showed that the shorter the frequency band of FM pulse, the better the enhancement with BP and WPD. The larger the upstream interval before the first pulse, the better the enhancement with WF. The longer the CF pulse at high SNR, the better the enhancement with MBSS because it tends to spread the patterns over time. Meanwhile, the proposed method produced highly efficient enhanced spectrograms for all of the investigated calls.

The temporal and spectral resolutions of the spectrograms produced by the BP approach are of high accuracy, since it does not operate a post processing to the full range of the noisy spectrogram, but only rejects the band which estimated to be noise. This was not the case with (MBSS, WF, and WPD) which post process the spectrograms for enhancement, results in changing the temporal and/or spectral resolutions. In the meantime, the edge detection algorithm of the proposed method was able to preserve the sound pulses into their almost original temporal and spectral locations while processing the noisy spectrogram. This is very important issue for any further pattern recognition assignment based on the enhanced spectrogram.

As a future aspect to this research work, an investigation will be made to avoid the loss of weak patterns done through limiting the dynamic range of the spectrogram. Moreover, improving the original spectrogram generation by adapting the applied STFT settings, this in correspondence improves the enhanced spectrogram.

\section{ACKNOWLEDGMENTS}

All bioacoustics calls which have been used in this study are brought under written permission from Aviosoft Bioacoustics GmbH, Berlin, Germany.

\section{REFERENCES}

[1] Hussein, W. B., Hussein, M.A.\& Becker, T., (2009) "Application of audio signal processing in the detection of the Red Palm Weevil", Proceeding of European Signal Processing Conference EUSIPCO2009, Glasgow, Scottland, 24-28.09.2009, pp 1597-1601.

[2] Obrist, M. K., Boesch, R. \& Flückiger, P. F.,(2004) "Variability in echolocation call design of 26 Swiss bat species: consequences, limits and options for automated field identification with a synergetic pattern recognition approach”. Mammalia, Vol.68, No. 4, pp 307-322.

[3] O'shaughnessy, D., (1987) "Speech Communication: Human and Machine", Addison-Wesley Publishing Co., Reading, MA, 1987, pp 56 - 106.

[4] Mallawaarachchi, A., Ong, S. H., Chitre, M., \&Taylor, E, (2008) "Spectrogram denoising and automated extraction of the fundamental frequency variation of dolphin whistles". J. Acoust. Soc. Am., Vol. 124, No. 2, pp 1-8.

[5] Oppenheim, Alan V., Schafer, Ronald W.\& Buck, John A. (1999). “Discrete-time signal processing”. Upper Saddle River, N.J., Prentice Hall, pp 468-471.

[6] Li, H., Zhang, Y. \&Xu, D., (2010) "Noise and Speckle Reduction in Doppler Blood Flow Spectrograms Using an Adaptive Pulse-Coupled Neural Network".EURASIP Journal on Advances in Signal Processing, pp 1-11. 
Signal \& Image Processing : An International Journal (SIPIJ) Vol.3, No.2, April 2012

[7] Ghanbari, Y., Karami, M. R.\&Amelifard,B., (2004) "Improved multi-band spectral subtraction method for speech enhancement", Proceedings of the 6th ISTED International conference SIGNAL AND IMAGE PROCESSING, pp225-230.

[8] Lampert, T. A. \& O'keefe, S.E.M, (2010) “A survey of spectrogram track detection algorithms". Applied acoustics, Vol. 71, pp 87- 100.

[9] Mellinger, D. K., (2002). Ishmael 1.0 User's Guide, Pacific Marine Enviromental Laboratory, Seattle, USA.

[10] Liu, W. A., Miller, R.C., Merzenich, K.D., \&Schreiner, C. E., (2003)“Acoustic variability and distinguishability among mouse ultrasound vocalizations,” J. Acoust. Soc. Am., Vol. 114, pp 34123422 .

[11] Ding, H., Soon, I.Y., Koh, S.N.\&Yeo, C.K., (2009) “A spectral filtering method based on hybrid Wiener filters for speech enhancement”. Speech Communication, Vol. 51, pp 259-267.

[12] Gur, B. M., \& Niezrecki, C., (2007). "Autocorrelation based denoising of manatee vocalizations using the undecimated discrete wavelet transform,” J. Acoust. Soc. Am., Vol. 122, pp 188-199.

[13] Ghanbari, Y.\&Karami, M.R., (2006)“A new approach for speech enhancement based on adaptive thresholding of wavelet packets' Speech communication, Vol. 48, pp 927-940.

[14] Chavan, M.S., Chavan, M.N., \&Gaikwad, M.S., (2010) "Studies on Implementation of Wavelet for Denoising Speech Signal”. International Journal of Computer Applications, Vol. 3, No.2,pp. 1-7

[15] Pandey, P.C., Pratapwar, S.S., \&Lehana, P.K., (2004) "Enhancement of Electrolaryngeal Speech by Reducing Leakage Noise Using Spectral Subtraction with Quantile Based Dynamic Estimation of Noise ".Proceeding of the $18^{\text {th }}$ international congress on acoustics ICA2004, pp. 3029-3032

[16] Sumithra, A., \&Thanushkodi, B., (2009) "Performance Evaluation of Different Thresholding Methods in Time AdaptiveWavelet Based Speech Enhancement”. IACSIT International Journal of Engineering and Technology,Vol.1,No.5,pp 42-51.

[17] Kamath, S.\& Loizou, P., (2002) "A Multi-band spectral subtraction method for Enhancing speech corrupted by colored noise", proceedings of ICASSP-2002, pp 37-43.

[18] Hermansky, H., Wan, E. A.\& Avendano, Carlos, (1994) "Noise Suppression in Cellular Communications", in Proceedings IEEE IVTTA'94, pp 85-88.

[19] Kim, H.G., Obermayer, K., Bode, M.\&Ruwisch, D., (2000) "real time noise cancelling based on spectral minimum detection and diffusive gain factors", in proceeding of $8^{\text {th }}$ Aust. Int. Conf. Speech Sci. and Tech., pp 250-255.

[20] Tuthill, T.A., Sperry, R.H. \& Parker, K.J., (1988) "Deviations from Rayleigh statistics in ultrasonic speckle," Ultrasonic Imaging, Vol. 10, No. 2, pp 81-89.

[21] Evans, N.D., Mason, J.S. \& Roach, M.J., (2002) "Noise Compensation using Spectrogram Morphological Filtering". In Proceeding of $4^{\text {th }}$ IASTED International Conference Signal Image Processes, pp 157-161.

[22] Steinberg, R. \&O'Shaughnessy,D., (2008) "Segmentation of a Speech Spectrogram using Mathematical Morphology”, in proceeding Acoustics, Speech and Signal Processing, ICASSP 2008, pp 1637 - 1640 .

[23] Gillespie D., (2004) "Detection and classification of right whale calls using an 'edge' detector operating on a smoothed spectrogram". Can Acoust 2004, Vol. 32, pp39-47.

[24] Lin, B.S., Wu, H.D., Chong, F.C.\&Chen, S.J., (2006) "Wheeze Recognition Based On 2d Bilateral Filtering Of Spectrogram”. Biomed Eng Appl Basis Comm, Vol. 18, pp 128-137.

[25] Dugnol, B., Fernández, C., Galiano, G. \&Velasco, J., (2007) "Wolves chorus noise reduction by spectrogram image processing", Signal Processing for Image Enhancement and Multimedia Processing, Springer Verlag.

[26] Ohtake, Y., Belyaev, A.G.\&Seidel, H.P., (2002) "Mesh smoothing by adaptive and anisotropic Gaussian filter". Proceeding of Vision, Modelling, and visualization 2002, pp 203-210. 
Signal \& Image Processing : An International Journal (SIPIJ) Vol.3, No.2, April 2012

[27] Hussein, W. B., Hussein, M.A.,\&Becker, T., (2010) "Detection of the Red Palm Weevil Using Its Bioacoustics Features." Journal of Bioacoustics, Vol. 19, No. 3, pp 177-194.

[28] Hussein, W. B., Moaty, A. A, Hussein, M. A.\& Becker, T., (2011) "A novel edge detection method with application to the fat content prediction in marbled meat". Pattern recognition, Vol. 44, No. 12, pp $2959-2970$.

[29] Eargle, J., (2005). “Handbook of recording engineering”, $4^{\text {th }}$ edition, Springer, pp 1-27.

[30] Deller, J.R., Hansen, J.H.L.\& Proakis, J.G., (2000) “Discrete-Time Processing of Speech Signals", second ed., IEEE Press, New York.

[31] Quackenbush, S.R., Barnwell, T.B.\& Clements, M.A, (1988) “Objective Measures of Speech Quality”. Englewood Cliffs, NJ, Prentice-Hall. 